\title{
PROFILE OF PATIENTS RECEIVING PALLIATIVE HOME CARE IN AN URBAN PRIMARY HEALTH UNIT IN THIRUVANANTHAPURAM - KERALA, INDIA
}

\section{Community Medicine}

Retheesh K H

Department of Community Medicine, Government Medical College,

Thiruvananthapuram.

Althaf A*

Department of Community Medicine, Government Medical College, Manjeri

*Corresponding Author

Princy S

Department of Community Medicine, Government Medical College,

Thiruvananthapuram.

Suni K Akbar

Department of Pediatric Nephrology, Government Medical College,

Thiruvananthapuram

\section{ABSTRACT}

Background: Community based approach to palliative care considers home based care as the corner stone of palliative care services. To ensure the services available and accessible to all, thrust should be given for a primary health care based approach. However there is an acute shortage of trained manpower. The primary health care physicians need to be trained in effective pain and symptom palliation. We aimed to study the clinical and socio-demographic profile of patients receiving palliative home care which would be helpful for the training of more man power and effective planning in this interdisciplinary endeavor. Methods: Cross sectional study among 345 participants who were registered with the palliative care unit at the Urban Primary Health Care Unit in Thiruvananthapuram, Kerala. Statistical Analysis: The data were entered using Microsoft excel for Windows XP and analyzed using SPSS 16.0 (Statistical Package for Social Sciences; SPSS Inc., Chicago, IL, USA). Results: Mean age was 70.9 years and $56.8 \%$ were females. $98 \%$ of the patients have care givers and $50 \%$ caregivers were elementary educated and $25 \%$ caregivers were employed. 51\% participants have benefits of pension schemes and others did not receive any kind of financial assistance. $70.7 \%$ did not have any kind of insurance coverage. $84.3 \%$ participants availed services of public hospitals. Majority had Hypertension (70.4\%) followed by diabetes mellitus (49.3\%) and dyslipidemia (26.7\%) as comorbidities. Conclusions: Contrary to the existing conventional expensive hospital care model, LSG supported home-based palliative care conducted by local primary health care system to certain extend can give comprehensive health care to bed ridden patients and thus improve the quality of life. The interface between institutional care and home care needs more exploration and prospective studies.

\section{KEYWORDS}

\section{Home-based Palliative Care, Urban Primary Health Care, Palliative Care Unit, Palliative Care Policy}

\section{Introduction and Background}

Palliative care is the prevention and relief of suffering of patients and their families facing the problems associated with life-threatening illness (1). These include physical, psychological, social and spiritual suffering of patients and psychological, social and spiritual suffering of family members. Palliative care increases feelings of psychological wellbeing, self-worth and self-esteem, reduces social isolation for dying people, and helps to normalize suffering. Earlier it was focused on patients with advanced cancers; the concepts have changed a lot at present. The home-based or hospital-based palliative care now benefits people with non-cancer chronic and life-limiting conditions. The World Health Organization has recognized that palliative care is an ethical responsibility of health systems and that integration of palliative care into public health care systems is essential for the achievement of the Sustainable Development Goal on universal health coverage (2). However there is an acute shortage of trained manpower. The primary health care physicians need to be trained in effective pain and symptom palliation. It is an interdisciplinary endeavor; the field includes medicine, nursing, social work, psychology, nutrition, and rehabilitation. The primary goal of palliative care is to optimize the quality of life of patients with advanced incurable diseases through control of physical symptoms and attention to the patient's psychological, social and spiritual needs.

The great majority of people who need palliative care prefer to remain at home, thus, it is medically and ethically necessary that palliative care be provided in the community, as part of primary health care. Primary health care providers with basic training in palliative care and symptom relief can respond effectively to most palliative care needs and arrange for transfer to a higher level of care when necessary. Emphasis should be given to continuity of care, respect for patients' values, equitable access to services, and attention not only to patients but also to their families. National Health Policy also envisages the provision of home based palliative care through frontline health workers (3).

The southern Indian state of Kerala has pioneered a community-based model of palliative care and was the first state to launch a palliative care policy in the country, in 2008 (4). It was the first time that any government in Asia has brought forth a policy in palliative care. It emphasized the community based approach to palliative care and considers home based care the corner stone of palliative care services, in which the local self-governments play the pivotal role. The policy highlighted the need for integrating palliative care with primary health care and disease specific treatment programs for effective coverage. The policy aimed to provide palliative care services for all individuals with the support of the society. The health system of the state will be improved to address the palliative care needs of the state. Kerala has been integrating palliative care with healthcare policy at all levels in a three-tier system. The policy was upgraded during 2019 to include neighborhood networks with trained staff that can identify patients' needs and provide home care at the primary level.

The palliative care activities formulated in consonance with the policy has been quite successful in initiating services with community participation in all parts of Kerala. The initiative aimed to identify patients in need, offer guidance for appropriate treatment, provide care at home for the bed-ridden and incurably ill and equip the primary health care system to provide care including medicines. Currently there are 908 palliative care units in the country, of which 841 units are based in Kerala. These shows the services are inaccessible to vast majority of the population in the country. To overcome the situation and to make the services available and accessible to all, thrust should be given for a primary health care based approach. In Kerala, with a population of 33 million and a crude death rate of 6.6 , around 80,000 dying patients and their families would be benefited each year.

Since it has been over a decade since the initiation of these activities, it is necessary to study the status of the program and the current needs of the patients. In this context, the following study was conducted to assess the clinical and socio-demographic profile of patients receiving Palliative home care and to enumerate the palliative care services provided by an urban primary health care unit in Kerala.

\section{MATERIALS AND METHODS}

The study was conducted during three months from September 2019 in the field area of Medical College Urban Health Center at Pangappara in Thiruvananthapuram District. The area has a population of $1,36,825$ in 
34,408 households. The cross sectional study was conducted by collecting information from all 345 patients enrolled and receiving home-based palliative care services. Census data was used for sampling purposes. The variables collected include sociodemographic, clinical diagnosis, associated co-morbidities and the services provided by palliative care unit. A pre tested semi structured questionnaire was used for data collection.

House to house survey was carried out to collect the required information. The investigator accompanied the palliative care team during their monthly home visits, which included a doctor, palliative care nurse and volunteers. After obtaining an informed consent, the interviewer gathered all the information required. The data collection was done along with regular follow-up services. Sufficient privacy was ensured while interviewing, in order to avoid respondent bias which might happen if they were interviewed in the presence of other family members. Those who were not willing to give consent and or severely ill patients who did not have family caregivers were excluded from the study. The protocol of the study was approved by the Institutional Research Committee and Institutional Ethics Committee of Government Medical College Trivandrum and written informed consent was collected from all subjects and those who are not able to give consent, consent was taken from their family care takers.

\section{Statistical analysis:}

The data were entered using Microsoft excel for Windows XP and analyzed using SPSS 16.0. The categorical variables will be expressed as proportions and quantitative variables as mean and standard deviation.

\section{RESULTS}

Mean age of the participants was 70.9 years. Among the 345 participants enrolled for the study $196(56.8 \%)$ were females. 338 were having family care givers and 7 were without care givers. 120 (34.4\%) subjects were taken care by their daughters and $87(25.3 \%)$ by wives. $50.3 \%$ of caregivers had at least high school education and only three were lacking formal education. The educational status of the caregivers may influence the quality of care received by the patient. Among the caregivers only 93 (25.5\%) were employed.

Table 1 - Disease conditions of participants

\begin{tabular}{|c|c|c|}
\hline Diagnosis & Frequency & Percentage (\%) \\
\hline Hemiplegia & 130 & 37.7 \\
\hline Paraplegia & 17 & 4.9 \\
\hline Quadriplegia & 4 & 1.2 \\
\hline TB & 5 & 1.4 \\
\hline Dementia & 12 & 3.5 \\
\hline Psychiatric disorder & 10 & 2.9 \\
\hline Parkinsonism & 18 & 5.3 \\
\hline Amputation of Limbs & 10 & 2.9 \\
\hline Diabetic foot & 5 & 1.4 \\
\hline Fracture Femur & 7 & 2 \\
\hline Cancer & 22 & 6.5 \\
\hline CAD & 20 & 5.8 \\
\hline CKD & 5 & 11.4 \\
\hline CLD & 1 & 0.3 \\
\hline COPD & 7 & 2 \\
\hline Arthritis & 6 & 1.7 \\
\hline Geriatric ailments & 33 & 14.4 \\
\hline Others & 11 & 3.2 \\
\hline Total & 345 & 100 \\
\hline
\end{tabular}

Among the study participants 177 (51.3\%) were beneficiaries of various pension schemes with 109 receiving old age pension. 168 $(48.7 \%)$ did not receive any kind of financial assistance. 244 (70.7\%) did not have any kind of insurance coverage. $85(24.6 \%)$ were covered under Rashtriya Swasthya Bima Yojana (RSBY) and 14 (4.1\%) under Karunya Arogya Suraksha Padhathi (KASP). All patients were following modern medicine while seven of them also availed treatment from alternative systems, four from homeopathy and three from Ayurveda. 291 (84.3\%) patients primarily depended government hospitals for their health needs and the rest went to private hospitals.

Among the subjects, majority had hypertension (70.4\%) followed by diabetes mellitus (49.3\%) and dyslipidemia (26.7\%) as comorbidities. Coronary artery disease, chronic kidney disease, chronic obstructive pulmonary disease and hypothyroidism were also reported in a few.
Majority of subjects had a history of cerebro-vascular accident. 22 $(6.5 \%)$ participants were suffering from malignancies. Other conditions include benign prostatic hypertrophy (BPH), uterine prolapse, seizure disorder, poliomyelitis, filariasis and diabetic neuropathy. More than half of the patients $208(60.3 \%)$ registered were bedridden. There were $130(37.7 \%)$ persons suffering from Hemiplegia, 22 (6.5) \% with malignancies, 20 (5.8\%) with Cardio Vascular Diseases, 18 (5.3\%) with Parkinsonism, 17 (4.9\%) with Paraplegia and $12(3.5 \%)$ with Dementia. People with non-cancer conditions were substantially higher users of home based palliative care services over a long period of time. All subjects were receiving services including general checkup, blood pressure monitoring and required medicines. Other services were given according to the condition of the patient.

\section{Table 2 - Palliative services received by the participants}

\begin{tabular}{|c|c|c|}
\hline Services & Frequency & Percentage (\%) \\
\hline Medicines & 345 & 100 \\
\hline BP monitoring & 345 & 100 \\
\hline GRBS & 166 & 48.1 \\
\hline Wound care & 6 & 1.7 \\
\hline Catheter care & 41 & 11.9 \\
\hline Ryle's Tube care & 6 & 1.7 \\
\hline Colostomy care & 2 & 0.6 \\
\hline Air bed & 4 & 1.2 \\
\hline Wheel chair & 10 & 2.9 \\
\hline Walker & 11 & 3.2 \\
\hline Walking stick & 4 & 1.2 \\
\hline
\end{tabular}

\section{DISCUSSION}

The present study assessed the socio-demographic and clinical profile of patients and the clinical services received by them. All subjects were enrolled under the palliative home care project.

$56.8 \%$ were females and the mean age of all subjects was 70.9 years. A study conducted by Sushma Bhatnagar et al found that at the national level also more women are confined to bed than men, exacerbated by a life time of gender-based discrimination (5).

Chronic diseases such as cardiovascular diseases and cancers lead to greater morbidity. Majority of patients had history of previously diagnosed chronic diseases and this reflects the high morbidity low mortality phenomenon and the epidemiologic and demographic transition in the Kerala society.

\section{CONCLUSION}

The study looked at the health status of patients undergoing palliative care and services given to them through the home-based program and found that in a resource poor setting, most of their needs can be cost effectively met through home-based palliative care services.

The services could address most of the medical, psychosocial, and supportive needs of the patients and reduce their pain and symptoms. Among the bed ridden patients a small proportion developed pressure ulcers most of which were managed by home care takers and infection rate was found to be low with timely guidance from palliative care team Contrary to the existing conventional hospital care model, local self-government supported home-based palliative care through primary health care system can give comprehensive and cost effective health care to bed ridden patients. For the evaluation of the program, further follow-up studies are needed. The interface between institution-based care and home care needs more exploration and prospective studies.

\section{REFERENCES}

1. WHO Definition of palliative care/WHO Definition of palliative care for children Geneva: World Health Organization; 2002. http:// www. who. int/ cancer/ palliative/ definition/en/, accessed 17 March 2018

2. Sixty-seventh World Health Assembly resolution WHA67.19 on Strengthening of palliative care as a component of comprehensive care throughout the life course http://apps.who.int/gb/ebwha/pdf files/WHA67/A67 R19-en.pdf?ua=1

National Health Policy 2017 MoHFW, GoI https:// www. nhp. gov. in/ nhpfiles/ national health policy 2017.pd

4. Paleri AK. Showing the way forward: Pain and Palliative Care Policy of the Government of Kerala. Indian J Palliat Care [serial online] 2008 [cited 2020 Sep 26];14:51-4. Available from: http://www.jpalliativecare.com/text.asp?2008/14/1/51/41936

5. National Sample Survey Office (NSSO) | Ministry of Statistics and Program Implementation | Government Of India [Internet]. [cited 2019 Nov 22]. Available from http://mospi.nic.in/NSSOa 